\title{
Abductive Two-Dimensionalism: A New Route to the A Priori Identification of Necessary Truths
}

\author{
Stephen Biggs and Jessica Wilson
}

penultimate version, forthcoming in a Synthese issue on meta-ontology, Carlo Rossi, ed.

\begin{abstract}
Epistemic two-dimensional semantics (E2D), advocated by Chalmers (2006) and Jackson (1998), among others, aims to restore the link between necessity and a priority seemingly broken by Kripke (1972/1980), by showing how armchair access to semantic intensions provides a basis for knowledge of necessary a posteriori truths (among other modal claims). The most compelling objections to E2D are that, for one or other reason, the requisite intensions are not accessible from the armchair (see, e.g., Wilson 1982, Melnyk 2008). As we substantiate here, existing versions of E2D are indeed subject to such access-based objections. But, we moreover argue, the difficulty lies not with E2D but with the typically presupposed conceiving-based epistemology of intensions. Freed from that epistemology, and given the right alternative---one where inference to the best explanation (i.e., abduction) provides the operative guide to intensions---E2D can meet access-based objections, and fulfill its promise of restoring the desirable link between necessity and a priority. This result serves as a central application of Biggs and Wilson 2016 (summarized here), according to which abduction is an a priori mode of inference.
\end{abstract}




\section{Introduction}

Kripke (1972/1980) famously argues that the traditional link between necessity and a priority is unsustainable, on grounds that many necessary truths about individuals and natural kinds---e.g., those expressed by 'Hesperus is Phosphorus' and 'water is $\mathrm{H}_{2} \mathrm{O}$ '---can only be known a posteriori. Though these arguments are widely seen as compelling, at least two considerations support trying to reforge the traditional link. First, it remains intuitive to suppose that, since necessary truths are true in all possible worlds, our knowledge of such truths must be to some extent a priori. Second, much theory and practice (philosophical, legal, semantic, scientific, etc.) presupposes that we can know modal truths independently of, or at least antecedent to the end of, empirical inquiry. It would thus be good news, both intuitively and theoretically, if the traditional link between necessity and a priority could be to some considerable extent restored.

This is the promise of epistemic two-dimensional semantics (E2D), advocated by Chalmers (e.g., 2006) and Chalmers and Jackson (2001), among many others. ${ }^{1}$ E2D characterizes distinct aspects of meaning as distinct intensions, and maintains that access to certain intensions provides a basis for a priori knowledge of a wide range of modal truths, including a conditional such basis for the truths at issue in Kripke's discussion. Hence, to roughly prefigure the E2D strategy, while it is a posteriori that, e.g., water is necessarily $\mathrm{H}_{2} \mathrm{O}$, our access to appropriate intensions provides a basis for our knowing a priori that if water (or the watery stuff) is actually $\mathrm{H}_{2} \mathrm{O}$, then water is necessarily $\mathrm{H}_{2} \mathrm{O}$; as such, the a posteriori contribution to the former necessity claim is limited to discharging the antecedent of a conditional that is known a priori.

\footnotetext{
${ }^{1}$ We count any version of what Chalmers (2006a) calls 'two-dimensionalism' as a version of E2D. E2D, then, is more general than what Chalmers (2006a) calls 'epistemic two-dimensionalism'. We explain our choice of terms in footnote 9. In addition to his own earlier work, Chalmers (2006a) identifies Jackson (1998, 2004), Braddon-Mitchell (2004), Lewis (1994), and Wong (1996) as fellow two-dimensionalists. Others who we take to advocate relevantly similar views include, e.g., Peacocke (1993), Boghossian (1996), Henderson and Horgan (2000, 2001), and Gertler (2002). Chalmers (2006a) cites his own earlier work and Chalmers and Jackson (2001) as advocating the more specific epistemic two-dimensionalism---though Chalmers and Jackson focus less directly on semantics than on reductive explanation. Advocates of a two-dimensional semantic framework who don't endorse any version of E2D include Stalnaker (1978, 1999), Kaplan (1979, 1989), and Davies and Humberstone (1981). See Chalmers (2006a, 2004, but especially 2006) for discussion of and further references to various interpretations of this semantic (or meta-semantic, as the case may be) framework.
} 
Can E2D fulfill this promise? The most pressing reasons to think not stem from objections that, for one or other reason, we cannot access the requisite intensions from the armchair, in which case our access to these intensions cannot provide the basis for a priori knowledge, modal or otherwise. ${ }^{2}$ Although, in our view, familiar versions of E2D succumb to such access-based objections, we contend that the difficulty lies with the typically presupposed conceiving-based epistemology of intensions, not with E2D as such. Freed from that epistemology, and given the right alternative---one where inference to the best explanation (i.e., abduction) provides the operative guide to intensions---E2D can meet access-based objections, and fulfill its promise of restoring the link between necessity and a priority. ${ }^{3}$

Our plan is as follows. In $\S 1$ we sketch the general E2D strategy for restoring the necessity-a priority link. In $\S 2$ we present, first, the conceiving-based epistemology of intensions (CEI) favored by existing proponents of E2D, and second, our preferred abduction-based epistemology of intensions (AEI). In $\S 3$ we argue that E2D+AEI can overcome access-based objections that undermine E2D+CEI; in $\S 4$ we argue that E2D+AEI can offer more plausible characterizations of actual philosophical practice and disagreement than E2D+CEI. In §5-6 we anticipate and respond to two objections to E2D+AEI. Specifically, in $\S 5$ we respond to the objection that AEI cannot deliver the a priori knowledge that E2D requires, since (it is claimed) abduction is an a posteriori mode of inference; and in $\S 6$ we respond to the objection that AEI too often delivers the "wrong" results. In $\S 7$ we offer some concluding remarks.

Before proceeding, we pause for a few preliminaries.

- To start, following other discussions of E2D, we presuppose that it aims to do each of the following: (a) deliver a priori knowledge of semantic facts, which in turn provides a basis for (fairly expansive) modal knowledge; (b) base our knowledge of these semantic facts on our access to relevant intensions; and (c) explain the a priority of our knowledge of these semantic facts as a consequence of

\footnotetext{
${ }^{2}$ See, e.g., Wilson (1982), Block and Stalnaker (1999), Byrne and Pryor (2006), and Melnyk (2008).

${ }^{3}$ We use 'abduction' to label inference to the best explanation for the sake of stylistic simplicity, not because we take inference to the best explanation to be Pierce's abduction. For reasons to distinguish inference to the best explanation from Pierce's abduction see, e.g., Minnameier 2004, Campos 2011, Plutynski 2011, and Mackonis 2013.
} 
our specifically armchair access to relevant intensions. Throughout, the semantic facts at issue concern what various token expressions (terms, statements), or the concepts they express, refer to in various conditions (scenarios, worlds, scenario-world pairs); for example, a relevant semantic fact might be that a token use of 'water' refers to $\mathrm{H}_{2} \mathrm{O}$ in the scenario that represents the actual world.

Melnyk describes and emphasizes the banality of the previous presuppositions in aprioristic semantic theorizing of the sort at issue in E2D (2008, pp. 267-268, our italics, but note his citations):

[C]onceptual or linguistic analysis [is] understood as ... settling into an armchair and asking oneself, for a variety of hypothetical situations, whether one would apply a given concept, or word, to something in that hypothetical situation ... the method is taken to be, together with further reflection, a sufficient means [for] the discovery a priori of necessary truths that can be formulated by using the given concept or word ... If conceptual analysis is a viable philosophical methodology, then there must be some account, even if it's very abstract, of what's going on in our minds when we use the method of hypothetical cases, some account which explains how in principle the method is capable of yielding a priori knowledge of necessary truths ... we have to assume that any such account will construe the necessary truths discovered by the method of hypothetical cases as semantic in origin, and then insist on the a priori knowability of the relevant semantic facts (see, for example, Peacocke 1993; Boghossian 1996; Henderson and Horgan, 2000 and 2001; Chalmers and Jackson 2001, especially 320-28; Gertler 2002).

These presuppositions about E2D illuminate talk of the 'epistemology of intensions' and 'knowledge of intensions', which we engage in below. Such talk is natural, given that E2D aims to base our knowledge of semantic facts on our access to intensions, and moreover to explain the a priority of our knowledge of semantic facts as a consequence of our specifically armchair access to intensions. In any case, if one is concerned that, insofar as intensions are functions, not claims (statements, propositions), intensions cannot be the proper content of knowledge or proper target of an epistemology, one can rather take such talk to be shorthand for talk of knowledge of the semantic facts determined by intensions. More generally, since our case that E2D+AEI is superior to E2D+CEI does not turn on this account of the semantic facts at issue in E2D, one can replace this account with any alternative that would allow E2D to fulfill its broad promise of restoring the traditional link between necessity and a priority (to some considerable extent), and then take us to argue that advocates of E2D should adopt AEI, rather than CEI, with respect to those facts---see footnote 5 for relevant discussion.

- Next, again following other discussions of E2D (and of conceptual analysis more generally), we 
presuppose that whether we can have a priori knowledge of the semantic facts at issue does not turn on whether we need experience to become minimally competent with certain expressions or associated concepts. Our use of "armchair access to intensions", "a priori knowledge of semantic facts", and related locutions should be understood accordingly. Moreover, in $\S 4$ we discuss in some detail what sort of experience, beyond that needed just to gain minimal competence with expressions or concepts, is or is not compatible with some knowledge's being a priori.

- Next, again following other discussions of E2D, we presuppose that E2D aims to show only that access to intensions provides a basis for a priori knowledge of conditionals, which in turn provide a basis for knowledge of necessary a posteriori truths, not to show that such truths are known either a priori or not at all.

- Next, again following other discussions of E2D, we presuppose that given the aforementioned reasons to restore tradition, the primary link of interest for us is that from necessity to a priority, not that from a priority to necessity. We focus, therefore, on the necessary a posteriori, not the contingent a priori--though, of course, some versions of E2D offer accounts of the contingent a priori. For ease of presentation, we focus more specifically on a posteriori necessities that involve natural kinds (e.g., water is $\mathrm{H}_{2} \mathrm{O}$ ).

- Finally, the interest of our discussion in what follows doesn't depend on endorsing E2D. Since AEI constitutes a new account of semantic analysis, on which such analysis proceeds by way of abduction, and since what we say about the epistemology of the intensions of expressions extends easily to the epistemology of the content of concepts, AEI constitutes, mutatis mutandis, a new account of conceptual analysis. Hence much of what we say here should engage anyone interested in semantic or conceptual analysis. (For example, one can see in AEI a potential resolution of the paradox of analysis.)

\section{Epistemic two-dimensional semantics}


Motivated by cases of cognitive informativity, Frege (1918/1997) distinguishes sense and reference as different aspects of meaning. In his favored example, someone taking 'Hesperus' and 'Phosphorus' to refer to the first heavenly body seen in the evening and morning, respectively, might learn that these words co-refer. Since this would be to learn something about the meaning of these words, reference is one aspect of meaning. But since someone can understand these words, to some extent, without knowing that they co-refer, there is also a second aspect of meaning---namely, sense.

Frege thinks of sense as intimately connected with a priority. Though there is some unclarity and associated controversy about exactly how he understands this connection, one can read Frege as suggesting that whether a claim is knowable a priori or not depends entirely on the nature of the relations among the senses of its constituent expressions and the nature of our access to these senses and relations. So, for example, on Frege's view 'bachelors are male' is knowable a priori because the senses of 'bachelor' and 'male' "overlap" in the right way and one can "grasp” the senses of 'bachelor' and 'male' in the right way. Though it is unclear, in particular, exactly what Frege takes to constitute the overlap and grasp at issue, we can say this much: on his picture, senses and their relations are accessible from the armchair, and this access provides a basis for any a priori knowledge that we are in position to have.

E2D refines Frege's picture, so understood, positing aspects of meaning that correspond roughly to sense and reference. ${ }^{4}$ Following Carnap (e.g., 1947/1950), each aspect either is or is represented by an intension, where intensions are roughly functions from possible worlds to extensions. More carefully, and now following Chalmers (e.g., 2006) in particular, intensions are functions from either scenarios or scenario-world pairs to extensions---where a scenario is either a centered world (i.e., one incorporating a subject's perspective) or a maximal set of sentences that is a priori consistent for anyone having our concepts and an idealized ability to discover their relations (and where a set is maximal if, modulo indeterminacies, for any sentence $S$, the set includes, directly or by implication, either $S$ or $\sim S$ ). The primary intension of an expression $E$, corresponding roughly to sense, takes each scenario $s$ to the

\footnotetext{
${ }^{4}$ As per considerations highlighted by Perry (2001), there may be many aspects of meaning, reflecting variations in what's held fixed in different uses of an expression. The two-dimensional semantic framework, including E2D, can be generalized accordingly.
} 
extension of $E$ in $s$ on the supposition that $s$ is actual. The secondary intension of an expression $E$, corresponding roughly to reference, takes each scenario-world pair $<s, w>$ to the extension of $E$ in $w$ given that $s$ is actual. Whether some, many, or all expressions are associated with both intensions is disputed; E2D requires that (at least) many natural kind expressions of the sort entering into a posteriori necessities are so associated.

There are a number of useful ways of characterizing these intensions. On one, primary intensions track extensions in contexts of utterance (centered worlds), and secondary intensions track extensions in contexts of evaluation (possible worlds), given a context of utterance (a particular centering within our world). On another, primary intensions track extensions in 'worlds considered as actual' (epistemic possibilities), and secondary intensions track extensions in 'worlds considered as counterfactual' (metaphysical possibilities), holding fixed which world is actual.

While these comparisons are each illuminating, what matters for us is this: on E2D, primary and secondary intensions correspond to what we might call, more specifically, epistemic sense and metaphysical reference. Epistemic sense is an aspect of meaning that can be accessed from the armchair, i.e., can be known a priori, and metaphysical reference is an aspect of meaning that, for many expressions, can be accessed only through additional experience, i.e., can be known only a posteriori. Correspondingly, primary intensions encode what any suitably competent speaker can know about the extensions of her expressions from the armchair, and secondary intensions encode an aspect of meaning which sometimes depends on how things are at whatever world is actual (in a way that precludes complete armchair access), and which may enter into certain necessary truths.

How do primary and secondary intensions assist in (re)forging the link between a priority and necessity? Doing this requires connecting the intensions, so that one who knows the primary intension of some relevant (e.g., natural kind) expression can infer something significant about its secondary intension---hence about necessity. But even an idealized subject cannot know a priori that water is in fact $\mathrm{H}_{2} \mathrm{O}$, as is required to know the secondary intension of 'water'. What, then, can one know a priori about secondary intensions, metaphysical reference, and necessity? 
Thinking in terms of a two-dimensional matrix assists in understanding how E2D answers this question (cf. Chalmers 2006a, following Stalnaker 1999), as follows:

\begin{tabular}{|l|l|l|l|}
\hline & $\mathrm{H}_{2} \mathrm{O}$-world & XYZ-world & \\
\hline $\mathrm{H}_{2} \mathrm{O}$-scenario & $\mathrm{H}_{2} \mathrm{O}$ & $\mathrm{H}_{2} \mathrm{O}$ & $\cdots$ \\
\hline XYZ-scenario & $\mathrm{XYZ}$ & $\mathrm{XYZ}$ & $\cdots$ \\
\hline$\cdots$ & $\cdots$ & $\cdots$ & $\cdots$ \\
\hline
\end{tabular}

The desired link between a priority and necessity (hence the justification for drawing the matrix as involving overlapping primary and secondary intensions) is then forged via three suppositions:

- First, we have in-principle armchair access to the space of scenarios---that is, to what worlds might have been actual, so to speak---represented in the leftmost column of the matrix.

- Second, we have in-principle armchair access to the extension of any token expression at any scenario on the hypothesis that the scenario is actual. In other words, we can have a priori knowledge of primary intensions, represented by the diagonal of the matrix.

- Third, we have in-principle armchair access to what the extension of any token expression would be at any world, on the hypothesis that any given scenario is actual. In other words, for any scenario $s$ and expression $E$, we can have a priori knowledge of what the secondary intension of $E$ would be, if $s$ were actual. More generally, we can have a priori knowledge of what we call generalized secondary intensions (and Chalmers calls two-dimensional intensions).

Since generalized secondary intensions are central to E2D, it is worth contrasting them with ordinary secondary intensions. The secondary intension of any token expression $E$ takes as input the actual world and any possible world $w$, and gives as output the extension of $E$ at $w$. The generalized secondary intension takes as input any scenario $s$ and any possible world $w$, and outputs the extension of $E$ at $w$ on the hypothesis that $s$ is actual. For example, the secondary intension of an appropriate token of 'water' takes as input the actual world (where the watery stuff is $\mathrm{H}_{2} \mathrm{O}$ ) and any world $w$, and returns the extension 
of 'water' at $w$---i.e., $\mathrm{H}_{2} \mathrm{O}$; the generalized secondary intension takes as input any scenario (e.g., a scenario in in which the watery stuff is XYZ), and any world $w$, and returns the extension of that use of 'water' at $w$ on the assumption that $s$ is actual, in this case XYZ. One might say that generalized secondary intensions track extensions in worlds that are counterfactual relative to a given scenario considered as actual, whether or not that scenario corresponds to the actual world.

Given these suppositions, a priori knowledge of intensions provides a basis for knowledge of a wide range of modal truths. As per the first bulleted supposition, the leftmost column of the matrix (the space of scenarios) can be specified independently of experience. As per the second, the diagonal of the matrix (the primary intension) can be specified independently of experience, indicating that "epistemic necessity" and a priority are deeply linked. As per the third, the remaining cells in the matrix (which fill in the generalized secondary intensions) can be specified independently of experience, indicating that metaphysical necessity and a priority are also deeply linked.

How significant is this final link? In knowing the primary intension of 'water', one knows a series of conditionals: if the watery stuff is actually $\mathrm{H}_{2} \mathrm{O}$, then 'water' refers to $\mathrm{H}_{2} \mathrm{O}$ in the actual world; if the watery stuff is actually XYZ, then 'water' refers to XYZ in the actual world; and so on. ${ }^{5}$ In knowing the generalized secondary intension of 'water', one knows another series of conditionals: if 'water' refers to $\mathrm{H}_{2} \mathrm{O}$ in the actual world, then 'water' refers to $\mathrm{H}_{2} \mathrm{O}$ in all worlds; and so on for XYZ, etc. From knowledge of these intensions, one can deduce a third set of conditionals: if the watery stuff is actually $\mathrm{H}_{2} \mathrm{O}$, then 'water' refers to $\mathrm{H}_{2} \mathrm{O}$ in all worlds; and so on for XYZ, etc. One who knows (through experience) that the watery stuff is actually $\mathrm{H}_{2} \mathrm{O}$ can thus use a priori knowledge of two intensions (primary and generalized secondary) to infer that water is necessarily $\mathrm{H}_{2} \mathrm{O}$. The only essential role for experience in knowing this a posteriori necessity lies in experience's being required to discharge the

\footnotetext{
${ }^{5}$ One who prefers a different account of the semantic facts at issue in E2D could substitute the following for the above conditionals without impacting the dialectic here: 'if the watery stuff is actually $\mathrm{H}_{2} \mathrm{O}$, then all samples of water are samples of $\mathrm{H}_{2} \mathrm{O}$ in the actual world'; 'if the watery stuff is actually XYZ, then all samples of water are samples of XYZ in the actual world'; and so on. Notice that 'water' no longer occurs inside quotes in the consequent of these conditionals, but the conditionals themselves are now (token) sentences, and hence, inside quotes. With some changes in form, we could even leave aside language, substituting appropriate propositions for these sentences (e.g., by removing the quotes from the above sentences)---with the result that E2D would deliver knowledge of the content of concepts/propositions, rather than the intensions of expressions.
} 
antecedent of a conditional (encoded by the primary intension) that is known a priori. Generalizing, it appears that with the exception of knowledge about which scenario corresponds to the actual world, everything about modality can be known a priori, on broadly semantic grounds. So, given knowledge of which world is actual, any suitably competent speaker can, in principle, infer all necessary truths, including necessary a posteriori truths, from a priori, broadly semantic knowledge. ${ }^{6,7}$

This, schematically, is how E2D aims to restore the traditional link between necessity and a priority.

The restoration is not perfect, since it remains that some necessary truths cannot be known without a posteriori knowledge of which world is actual. The restoration is nonetheless significant, however, since it provides a post-Kripkean basis for gaining a priori knowledge of a wide range of modal truths, revealing everything about modality to one who knows which world is actual, and much about modality even to one not in possession of the latter information. ${ }^{8,9}$

\section{Two epistemologies of intensions}

The above characterization of E2D leaves open how one accesses intensions from the armchair, thereby

\footnotetext{
${ }^{6}$ There may be disagreement about which conditionals the primary intension encodes, of course. Perhaps the primary intension for some appropriate use of 'water' encodes conditionals relevant to scenarios in which the most common clear, drinkable liquid in the actual world is half water and half XYZ, or is wildly disjunctive (see Bealer 2002, p. 109 for relevant discussion, though he's no fan of E2D). There are limits, however. One cannot hold that an ordinary use of 'water' refers to whatever is at the beginning of the causal chain that led to one's acquisition of 'water', since 'water' does not refer, e.g., to an evil demon, even if the chain begins with such an entity-though see Chalmers (2005) for (at least inspiration for) an opposing view.

${ }^{7}$ Our three suppositions correspond to four of what Chalmers (2006a, p. 585) calls the "[f]ive core claims of twodimensionalism" - specifically, to his first, third, fourth, and fifth theses. We are neutral as regards his second core claim, which identifies a compositional relationship between complex and simple expressions, but see no reason to exclude this claim here.

${ }^{8}$ Note that to know which world is actual is not thereby to know the extension of every token expression at the actual world. For example, scenarios understood as sets of sentences include only semantically neutral expressions, where, informally, an expression is semantically neutral if its extension in scenarios considered as counterfactual does not depend on which scenario is considered as actual (cf., Chalmers 2006, p. 87). No ordinary use of 'water' is semantically neutral, since whether it refers to $\mathrm{H}_{2} \mathrm{O}$ or XYZ in scenarios considered as counterfactual depends on which scenario is considered as actual. As such, to entertain a scenario $s$ is not thereby to know the extension of 'water' at $s$, even if one can know the extension of 'water' at $s$ a priori. Most names, natural kind words, and indexical words resemble 'water' in this respect. By contrast, many token expressions plausibly are semantically neutral. Chalmers offers ordinary uses of 'and', 'philosopher', 'friend', 'consciousness', and 'cause' (p. 86); other possible candidates include 'lake', 'stream', 'clear', 'potable', ' $\mathrm{H}_{2} \mathrm{O}$ ', and 'XYZ'. Scenarios trivially include only these sorts of expressions.

${ }^{9}$ We pause to explain our decision to use the label 'epistemic two dimensional semantics', rather than either 'twodimensionalism' or 'epistemic two-dimensionalism'. Chalmers (2004, p. 166) distinguishes "epistemic" from "contextual" interpretations of two dimensional semantic frameworks, maintaining, as we agree, that only epistemic interpretations can fulfill E2D's promise of restoring (to any considerable extent) the traditional link between necessity and a priority. Since we are interested only in interpretations that can fulfill this promise, we are interested in specifically epistemic interpretations; hence our moniker includes the qualifier 'epistemic'. And since we are exploring epistemic interpretations of two-dimensional semantic frameworks, we use the label 'epistemic two dimensional semantics'; this designation moreover serves the purpose of distinguishing our use from the specific view that Chalmers (2006a) calls 'epistemic two-dimensionalism', which use invokes (what we take to be) a misguided (conceiving-based) epistemology of intensions.
} 
acquiring a priori knowledge of intensions---that is, leaves open the epistemology of intensions. How, exactly, is one supposed to gain this knowledge? To say, as proponents of E2D commonly do, that a priori deliberation involves 'conceptual analysis' or 'idealized rational reflection' does not go much further in elucidating the operative process. In what follows, we first motivate the claim that standard versions of E2D more specifically implement a conceiving-based epistemology of intensions (at least insofar as they presume a particular epistemology of intensions), next sketch our abduction-based alternative, then briefly compare the two.

Among advocates of E2D, Chalmers (in various works) and Chalmers and Jackson (in their 2001) say the most about how the conceiving at issue works. Accordingly, we focus on their work while describing a conceiving-based epistemology of intensions (CEI). We expect our concerns about CEI to apply, mutatis mutandis, to the epistemology of intensions operative in any existing version of E2D.

To be clear, Chalmers and Jackson (2001) discuss conceiving qua method for identifying the content of concepts/thoughts, not qua method for identifying the intensions of terms/statements. Nonetheless, we use their 2001 to characterize the conceiving at issue in CEI, since what they say about the role of conceiving and rational reflection in investigating the content of concepts/thoughts precisely parallels what they say elsewhere about the role of conceiving and reflection in investigating the intensions of terms/statements; and since, moreover, Chalmers (2006a, p. 588) cites Chalmers and Jackson (2001) as advocating E2D, which suggests that he intends their discussion of the epistemology of content to transfer to the epistemology of intensions. Because Chalmers and Jackson focus on the content of concepts/thoughts, rather than on the intensions of terms/statements, when we use their work to elucidate the conceiving at issue in CEI, and more generally when we compare our thoughts on the a priority of abduction to their thoughts on the a priority of conceiving, we shift freely between discussing, e.g., the extensions of concepts/thoughts and the extensions of terms/statements.

\subsection{A conceiving-based epistemology of intensions (CEI)}

It is natural to assume that rational reflection and a priority go hand in hand. As Chalmers elaborates, "a 
sentence token is a priori when it expresses an a priori thought", where an a priori thought is one that "can be conclusively non-experientially justified on idealized rational reflection" (2006, p. 98). Here to be conclusively non-experientially justified is to be indefeasibly justified by idealized rational reflection alone; but how exactly are we to understand rational reflection and its idealized counterpart, applied, in particular, to the conditionals at the heart of the E2D strategy? Relatedly, what exactly is involved in idealized "conceptual analysis" or "a priori entailment" (as these are seen by, e.g., Chalmers and Jackson 2001)?

Answers from proponents of E2D tend to be more specific about what rational reflection (or variations on this theme; we will not carry all these forward) is not than about what it is. Hence, Chalmers and Jackson (2001) start by noting that one may reason about a Gettier case (as antecedent) to the rejection of the application of the concept of knowledge (as consequent) even in the absence of an explicit definition of knowledge, saying, "This pattern, whereby a conditional ability to evaluate a concept's extension yields elucidation of a concept without a finite counterexample-free analysis, is illustrated very clearly in the case of 'knowledge'" (p. 323); and they repeatedly maintain that in this case as well as those involving natural kinds that are more typically the target of the E2D strategy, "empirical information plays no essential role in justifying belief in [the associated] conditionals (p. 321; see also p. 323, p. 325, p. 333, and elsewhere). But what exactly is the "conditional ability" in question? They go on to say:

Once an essential role for explicit definitions is eschewed, the model of conceptual analysis that emerges is something like the following. When given sufficient information about a hypothetical scenario, subjects are frequently in position to identify the extension of a given concept, on reflection, under the hypothesis that the scenario in question obtains. Analysis of a concept proceeds at least in part through consideration of a concept's extension within hypothetical scenarios, and noting regularities that emerge. [...] What emerges as a result of this process may or may not be an explicit definition, but it will at least give useful information about the features in virtue of which a concept applies to the world. [...] The possibility of this sort of analysis is grounded in the following general feature of our concepts. If a subject possesses a concept and has unimpaired rational processes, then sufficient empirical information about the actual world puts a subject in a position to identify the concept's extension. (p. 322)

But what exactly is it to engage in "consideration of a concept's extension", or to be "in a position to identify [a] concept's extension"? Here, one might recall Melnyk's suggestion that "conceptual analysis is a viable philosophical methodology" only if there is "some account, even if it is very abstract, of what's 
going on in our minds when we use the method of hypothetical cases" (2008, pp. 267-268).

Other broadly gestural characterizations include that "sufficient information about an epistemic possibility enables a subject to know what a concept's extension will be [in that scenario]" (p. 323), that "possession of a concept such as 'knowledge' or 'water' bestows a conditional ability to identify the concept's extension under a hypothetical epistemic possibility, given sufficient information about that epistemic possibility and sufficient reasoning" (p. 324); that "a priori conditionals will reflect the way in which we can identify a concept's referent" (p. 325); that "if we possess a concept, then sufficient empirical information $E$ enables us to conclusively identify the concept's extension and to know associated truths $\mathrm{C}[\ldots]$ whether the information in $E$ is actual or hypothetical (p. 325); that relevant nontrivial information about the scenario in the antecedent is "sufficient [...] for determining the truth" of a claim involving the concept in the consequent (p. 333); that given the relevant non-trivial information about the scenario, we can "process this information on a priori grounds" to "ascertain the truth of" a claim involving the concept in the consequent (p. 335). To our mind, none of these gestures do much to positively characterize the intended methodology.

Still, given certain specified commitments, a picture emerges on which the preferred epistemology of intensions involves conceivability, broadly construed, where the justificatory role of conceiving is assumed not to involve broadly abductive reasoning (hence it is that our proposed alternative $i s$ a genuine alternative to the standardly operative epistemology of intensions). We raise to salience three considerations in support of this claim.

To start, that conceivability is what is ultimately at issue is supported by Chalmers's (2002) defense of conceivability as entailing possibility. Here Chalmers also expands on the notion of conceivability, noting that it may be positive or negative, and prima facie or idealized; and he clarifies that for purposes of conclusively justifying beliefs about intensions and associated modal claims, what is crucially at issue is idealized positive conceiving, understood as involving a kind of rational perceiving or 'modal imagining':

Positive notions of conceivability require that one can form some sort of positive conception of a situation in which $\mathrm{S}$ is the case. One can place the varieties of positive conceivability under the broad rubric of imagination: to positively conceive of a situation is to in some sense imagine a 
specific configuration of objects and properties [...] Different notions of conceivability correspond to different notions of imagination. One such notion is tied to perceptual imagination. A subject perceptually imagines that $\mathrm{S}$ when the subject has a perceptual mental image that represents $\mathrm{S}$ as being the case. [...] There is a sense in which we can imagine situations that do not seem to be potential contents of perceptual experiences [e.g.,] molecules of $\mathrm{H} 2 \mathrm{O}$, or Germany winning the Second World War [, or] the existence of an invisible being that leaves no trace on perception. [...] In this case, we have an intuition of (or as of) a world in which S, or at least of (or as of) a situation in which $\mathrm{S}$, where a situation is (roughly) a configuration of objects and properties within a world. We might say that in these cases, one can modally imagine that $\mathrm{P}$. One modally imagines that $\mathrm{P}$ if one modally imagines a world that verifies $\mathrm{P}$, or a situation that verifies P. (pp. 150-151)

Here we have a positive characterization of the intended methodology, in at least certain of its applications. But, however exactly conceiving is understood, there are two clear reasons to think that abductive inference is not any part of conceiving. First is that Chalmers, Jackson, and other familiar proponents of E2D take the products of conceivability to be conclusive or indefeasible. As we will argue in $\S 5$, there is a case to be made that abductive reasoning is properly understood as a priori, but is inconclusive nevertheless. Second, Chalmers and Jackson are explicit—when arguing, in particular, against Block and Stalnaker's (1999) claim that the justification for the conditional claims at the heart of E2D might rely on broadly abductive considerations (e.g., simplicity) — that in their view abductive considerations do not play any 'essential justificatory' role in the cases at hand. While all parties to that particular dispute assume (incorrectly, in our view) that abduction is an a posteriori mode of inference, ${ }^{10}$ independent of this issue Chalmers's and Jackson's response makes clear that the standardly assumed epistemology of intensions underlying E2D is one that does not appeal to abduction nor to any of the inferential principles (of simplicity, parsimony, fruitfulness, etc.) standardly taken to underlie abduction (see especially Chalmers and Jackson, pp. 342-350). ${ }^{11}$

\subsection{An abduction-based epistemology of intensions (AEI)}

Our preferred epistemology of intensions---AEI---replaces conceiving with abduction. Abduction, as we

\footnotetext{
${ }^{10}$ Though Block and Stalnaker (1999) maintain that their argument that abductive principles play an ineliminable justificatory role in assessing claims about reduction stands even if abductive principles are "as a priori as you like" (29).

${ }^{11}$ One might wonder, as a referee did, if the open-texturedness of the operative notion of conceivability might stretch still further to allow all abductive considerations to enter into CEI. Perhaps, but if so, then Chalmers and Jackson, among other proponents of E2D, will have to retract not just their assumption that idealized rational reflection/positive conceivability results in conclusively justified beliefs or thoughts (hence will have to retract their usual reply to Block and Stalnaker), but also much of the argumentation they offer in response to access-based concerns with their view; for as we'll argue down the line, on an abductionbased epistemology of intensions, many of these concerns allow for responses much different from (and better than) those that Chalmers, Jackson, and others provide.
} 
understand it, proceeds by assessing the extent to which a range of candidate theories satisfies the (perhaps competing) dictates of various non-demonstrative inferential principles, including, for example, principles of ontological parsimony, explanatory comprehensiveness, and fruitfulness. Such principles are sometimes called theoretical virtues; we typically call them abductive principles. To use abduction when deciding among competing theories is to choose the theory that best explains some target (i.e., the explanandum), where the underlying abductive principles and their weightings determine how theories are ranked. This sketch leaves open exactly which principles and weightings are at issue. We leave certain details open both because they are not required to motivate our alternative epistemology of intensions, and because no specific set of principles or way of balancing them is uncontroversial; as will later become clear, this flexibility is a feature, not a bug, of our approach. ${ }^{12}$

What theories and explananda are at issue for primary intensions? The candidate intensions determine the theories, which are representations in propositional form of the intensions. Consider, for example, three such theories for an ordinary token of 'water'.

(i) In any scenario considered as actual, 'water' refers to the basis of life.

(ii) In any scenario considered as actual, 'water' refers to the watery stuff.

(iii) In any scenario considered as actual, 'water' refers to $\mathrm{H}_{2} \mathrm{O}$.

The first theory represents the intension that delivers the basis of life at any scenario it receives as input, regardless of what superficial or "deep" properties that basis has; hence in the scenario that represents the actual world, samples of that basis are samples of both (and only) watery stuff and $\mathrm{H}_{2} \mathrm{O}$, but in other scenarios they will be neither. The second represents the intension that delivers the watery stuff at any scenario it receives as input, regardless of the chemical composition of that stuff---in the scenario that represents the actual world, samples of that watery stuff are samples of (and only of) $\mathrm{H}_{2} \mathrm{O}$, but in other scenarios they might be samples of some other variety. The third represents the intension that delivers $\mathrm{H}_{2} \mathrm{O}$ at any scenario it receives as input. To choose among competing primary intensions for 'water' is to choose among theories such as (i)-(iii). These theories aim to explain, inter alia, what we would (or

\footnotetext{
${ }^{12}$ For more on abduction and abductive principles, see Lipton (1991/2004).
} 
should) think when contemplating various scenarios (e.g., what we would or should take the extension of an ordinary token of 'water' to be given various empirical results, how we would or should act if someone dying of thirst calls for 'water' in an XYZ-scenario, and so on).

How might abductive principles enter into ranking these theories? The third theory is in one respect less explanatorily comprehensive than the other two, since unlike these others, it cannot explain the thoughts or actions of those contemplating scenarios in which the watery stuff is actually XYZ (or anything different from $\mathrm{H}_{2} \mathrm{O}$ ). Of course, this is not a decisive case against (iii), let alone for (i) or (ii); still, it illustrates that abductive principles (in this case, a principle that captures the virtue of explanatory comprehensiveness) can play a role in assessing theories of primary intensions. ${ }^{13}$

What theories and explananda are at issue for generalized secondary intensions? Again, the candidate intensions determine the theories, which are just propositional representations of the intensions. Consider, for example, three such theories for an ordinary token of 'water'.

(i') In any world considered as counterfactual relative to any scenario considered as actual, 'water' refers to the basis of life.

(ii') In any world considered as counterfactual relative to any scenario considered as actual, 'water' refers to the watery stuff.

(iii') In any world considered as counterfactual relative to any scenario considered as actual, 'water' refers to the common underlying compositional structure of the watery stuff in the scenario considered as actual (e.g., in any world considered as counterfactual relative to an $\mathrm{H}_{2} \mathrm{O}$-scenario, 'water' refers to $\mathrm{H}_{2} \mathrm{O}$; in any world considered as counterfactual

\footnotetext{
${ }^{13}$ One might have the following concern: given AEI, our relevant thoughts and actions are the explananda on the basis of which we should choose among competing primary intensions for any given term; CEI instructs us to make that choice on the basis of our intuitive judgments; the thoughts and actions at issue given AEI just are (or are equivalent to) the intuitive judgments at issue given CEI; so, AEI and CEI will invariably deliver the same results. Against this concern, we respond: because abduction is ampliative where conceiving is not, abduction can, e.g., fill out indeterminate primary intensions where conceiving cannot (since abduction can deliver extensions for expressions at scenarios where conceiving cannot). It is primarily this difference in the mode of inference itself, rather than a difference between the "input" available to abduction versus that available to conceiving, that is crucial vis-à-vis primary intensions.
} 
relative to an XYZ-scenario, 'water' refers to XYZ; and so on). ${ }^{14}$

The first theory represents the intension that, for any scenario world pair $\langle s, w>$, delivers the basis of life at $w$, regardless what the basis of life is at the actual world. The second represents the intension that, for any scenario world pair $\langle s, w>$, delivers the watery stuff at $w$, regardless of the compositional structure that water has at $s$ or $w$. The third represents the intension that, for any scenario world pair $\langle s, w>$, delivers whatever at $w$ has the same underlying compositional structure as is had by the watery stuff at $s$. To choose among competing generalized secondary intensions for 'water' is to choose among theories such as (i')-(iii'). These theories aim to explain, among other things, that in the actual world all and only samples of water are samples of $\mathrm{H}_{2} \mathrm{O}$.

How might abductive principles enter into ranking these theories? The third theory arguably explains the actual coincidence of samples of water and samples of $\mathrm{H}_{2} \mathrm{O}$ in a more ontologically parsimonious way than the other two, since unlike these others, it is consistent with the claim that water and $\mathrm{H}_{2} \mathrm{O}$, qua natural kinds (however exactly kinds are understood---e.g., as properties) are identical.

To see this, first note that notwithstanding that applying the E2D strategy requires setting up the 2D matrix, so to speak, and hence contemplating extensions of expressions in or relative to other scenarios--that is, contemplating goings-on in or counterfactual to other worlds considered as actual---on the usual assumption that only one world is in fact actual, the associated semantic facts are considered to be epistemically, but not metaphysically, relevant. I.e., even if we can make sense of its having turned out that the actual world was one where 'water' referred to XYZ, this counter-actual semantic fact wouldn't be taken by proponents of the claim that water and $\mathrm{H}_{2} \mathrm{O}$ are identical kinds to undermine that claim. Hence so far as the metaphysics of the referent of 'water' is concerned, it suffices to attend only to (what is usually called) the secondary intension, tracking extensions in worlds considered as counterfactual relative to the scenario (world) that is (in fact) actual.

Keeping this latter point in mind, it is clear that of the three theories, only the third is compatible with

\footnotetext{
${ }^{14}$ Of course, some theories will be more difficult to express because the underlying candidate intension is more unruly-perhaps taking an $\mathrm{H}_{2} \mathrm{O}$-scenario and arbitrary world $w$ as input and delivering all and only watery stuff at $w$ as output, but taking an XYZscenario and $w$ as input and delivering all and only XYZ at $w$ as output.
} 
water's being identical with $\mathrm{H}_{2} \mathrm{O}$; for only this theory ensures that the referent of 'water' at counterfactual worlds, relative to the actual world scenario, is (as the kind identity requires) $\mathrm{H}_{2} \mathrm{O}$, whereas the other theories do not ensure this, since the (chemical) basis of life and the (chemical) composition of the watery stuff might be different at different counterfactual worlds, relative to the actual world scenario. Hence, while all three theories can explain the actual coincidence of water and $\mathrm{H}_{2} \mathrm{O}$, theory (iii') does so in the most ontologically parsimonious fashion. Of course, this is not a decisive case for (iii'), let alone against (i') or (ii'); still, it illustrates that abductive principles (in this case, a principle of ontological parsimony) can play a role in assessing theories of secondary intensions.

Finally, to be clear, this sketch is not meant to constitute instructions for using abduction to assess intensions, though some do reason along similar lines. ${ }^{15}$ Rather, it is meant to differentiate AEI from CEI while giving one a sense, however schematic, of how abduction can be used to identify intensions.

\subsection{Inferring ordinary truths}

Two versions of E2D are now on the table, E2D+AEI and E2D+CEI. We can further illuminate differences between them by considering how each treats inferences from base truths to ordinary truths. ${ }^{16}$ Why is semantics relevant to such inferences? When identifying the extension of an expression $E$ at a scenario $s$, one must begin with a description of $s$ which excludes $E$, but includes enough information to determine $E$ 's extension at $s$. Typically, this is accomplished by entertaining a description that uses expressions that are, in some relevant sense, more basic than $E$. Rather than involving 'water', for example, a description might involve 'clear', 'potable', 'hydrogen', etc. Accordingly, inferring ordinary truths from base truths corresponds rather precisely to identifying primary intensions, such that the epistemology for one transfers to the other. We can thus contrast E2D+CEI with E2D+AEI, qua epistemologies of primary intensions, by noting how each differently schematizes inferences from base truths to ordinary truths.

As per Chalmers (2006), let ' $D$ ' be a sentence expressing all base truths at a scenario $s$ (i.e., base

\footnotetext{
${ }^{15}$ See, e.g., Block and Stalnaker 1999 and Biggs (2011).

${ }^{16}$ Chalmers (2006) uses the labels base and ordinary truths for, roughly, fundamental and derivative truths respectively.
} 
claims that would be base truths if $s$ were actual), and ' $A$ ' be any sentence expressing an ordinary truth at $s$ (i.e., an ordinary claim that would be an ordinary truth if $s$ were actual). The inference from $D$ to $A$ can be schematized neutrally:

$$
\begin{aligned}
& D \\
& D \rightarrow A \\
\therefore & A \text { [modus ponens] }
\end{aligned}
$$

In step 1, one accepts $D$ as a working hypothesis. In step 2, one uses the epistemology of intensions to determine that $A$ follows from $D$. In step 3, one deduces $A$. E2D+CEI and E2D+AEI disagree about the justification for step 2: the former holds that step 2 is justified conclusively through conceiving; the latter holds that step 2 is justified inconclusively through abduction. Since each holds that inferences of this kind are always justified, the following schematizations for E2D+CEI (left) and E2D+AEI (right) are more perspicuous:

$$
\begin{array}{ll}
D & D \\
\therefore A \text { [conceiving] } & \therefore A \text { [abduction, appealing to abductive principles } \mathrm{a}_{1}, \mathrm{a}_{2}, \ldots \mathrm{a}_{\mathrm{n}} \text { ] }
\end{array}
$$

(We leave it to the reader to generate a similar contrast of E2D+CEI with E2D+AEI qua epistemologies of generalized secondary intensions.)

A key similarity between CEI and AEI is now clear: each offers an inferential principle. Two differences are also clear. First, AEI is methodologically broader than CEI, in incorporating the full spectrum of theoretical desiderata at issue in abductive deliberation. Second, AEI, unlike CEI, only aims to deliver inconclusive knowledge. Though this renders AEI-based knowledge of modal claims similarly inconclusive, so long as AEI typically tracks what intensions are in fact, the link between a priority and necessity will be substantially reforged. Indeed, as we will see next, it is thanks to both of these differences that E2D+AEI---abductive two-dimensionalism---delivers different results than E2D+CEI visà-vis both primary and secondary intensions, is able to overcome the access-based objections that undermine $\mathrm{E} 2 \mathrm{D}+\mathrm{CEI}$, and is able to make better sense of actual philosophical practice and disagreement than can E2D+CEI. 


\section{Access-based objections}

The most compelling challenges to E2D are due to various access-based objections, according to which we cannot, for one reason or another, have a priori knowledge of the primary (hence secondary) intensions supposed to provide a basis for our knowledge of modal truths. Here, we consider two of these objections, and argue that while each is pressing against E2D+CEI, neither is pressing against abductive two-dimensionalism. Along the way, we show that which primary intensions E2D delivers sometimes depends on which epistemology of intensions is operative. ${ }^{17}$

\subsection{Insuperable semantic indeterminacy}

According to the first access-based objection, there is more indeterminacy in natural kind predicates than E2D can tolerate. Recall that on E2D our a priori knowledge of primary and generalized secondary intensions together provides the basis for any modal knowledge that we have. Hence on E2D, modal knowledge is as sparse as (insuperable) indeterminacy is widespread. E2D aims to account for our having (in principle) knowledge of a wide range of modal truths, including necessary a posteriori truths about natural kinds. As such, widespread (insuperable) indeterminacy of natural kind predicates would undermine E2D.

We argue below that there is widespread indeterminacy in natural kind predicates, that cannot be overcome through conceiving alone. Our discussion is compatible with two understandings of the indeterminacy at issue. It could be that, while the natural kind predicates at issue determinately express a certain concept, many such concepts are indeterminate, in that they may not, in and of themselves (and given CEI), determine their application (or that of associated predicates) in the full range of scenarios. Alternatively, it could be that which of multiple completely determinate concepts a given natural kind predicate expresses is indeterminate. Nothing we say turns on which understanding one chooses.

\footnotetext{
${ }^{17}$ To be clear, we think that some objections to E2D do not hinge on which epistemology of intensions is operative, and can be overcome by E2D+CEI. We place objections from, e.g., Stalnaker $(1999,2001)$ and Soames $(2005)$ in this category. For responses to these objections that we take to succeed, see Chalmers $(2006, \S 4)$; for response to the latter in particular see Chalmers (2006b).
} 
One might worry that on either understanding, the claim that the intension for a term does not determine its extension at some scenarios implies that it has no primary intension, not, as we may seem to suggest, that it has a primary intension that we cannot access. We call indeterminacy-based objections access-based objections, nonetheless, in order to emphasize that the indeterminacy at issue only appears to be insuperable if one adopts the wrong account of how we access intensions; that said, nothing important turns on this label.

The case for widespread indeterminacy begins with Wilson's $(1982,2006)$ observation that our (idealized) application of natural kind predicates can be determined by arbitrary factors. In one of Wilson's toy cases, whether members of an isolated tribe put airplanes in the extension of 'bird' depends on whether the first airplane they encounter is overhead or on the ground. If such historical accident partly determines whether 'bird' applies to airplanes, then, Wilson reasonably assumes, the full range of the predicate's extension is not antecedently determined. Relatedly, although Wilson does not discuss this kind of case, ordering phenomena in Sorites series suggest that arbitrary factors can influence predicate application: the breaking point in applications of 'blue' may depend non-systematically both on where along the spectrum one starts and on psychological factors (cf. Raffman 1994).

In his early work, Chalmers (1996) says that such indeterminacy is "no problem" for E2D:

There may of course be borderline cases in which it's indeterminate whether a concept would refer to a certain object if a given world turned out to be actual. This is no problem: we can allow indeterminacies in a primary intension, as we sometimes allow indeterminacies in reference in our own world (p. 364).

Chalmers is right that E2D can tolerate some indeterminacy. But as above, it cannot allow that insuperable indeterminacy is widespread without undermining its raison d'etre of restoring the link between necessity and a priority.

Anticipating this difficulty in his 1996, Chalmers suggests that conceivers can eliminate indeterminacy from primary intensions by foreseeing relevant accidents. In Wilson's case, Chalmers says that one "might try to classify these two different scenarios [airplane first seen in the sky or on the ground, respectively] as different ways for the actual world to turn out, and therefore retain a fixed, detailed 
primary intension" (p. 364). On this strategy, the fully determinate primary intension of 'bird' includes planes in its extension if the tribe members first see a plane overhead, but not if they first see it on the ground. Either way, according to the suggestion, indeterminacy is resolved.

Chalmers's suggestion has traction against the objection only if conceivers can foresee how intensions are sensitive to accidents. But, as we see it, a deeper lesson of Wilson's case is that the influence of accidents cannot be foreseen, at least not by conceiving alone: determinism and such aside, those using conceiving alone might apply 'bird' differently even relative to the same historical facts. After all, there are any number of respects of dissimilarity between airplanes and birds, even when the former are in flight, and a minor difference in attention to these features might result in a different decision about whether 'bird' applies. We can register, post-hoc, extensions resulting from whatever decision was in fact made, but that does not show that anyone can antecedently identify these extensions and corresponding intensions through conceiving alone.

More recently, Chalmers (2012) accepts that such indeterminacy may be insuperable. In discussing Wilson's cases, he says it "is plausible" that "later extensions [of the kind of expressions at issue in Wilson's cases] depend on idiosyncratic developments, and verdicts about such cases are not determinately prefigured in a user's original use of an expression" (p. 231). In the context where he makes this concession, Chalmers is discussing his scrutability thesis, ${ }^{18}$ not E2D, and so he continues by noting that "widespread indeterminacy is quite compatible with the scrutability thesis" (p. 231). These are compatible, he thinks, because in cases where the predicate at issue is indeterminate, relevant statements including that predicate will themselves be indeterminate, rather than true or false, and so, such indeterminacy does not prevent one from deducing any ordinary truth at $s$; for example, at an appropriate scenario in which the native's never encounter a plane, their ordinary uses of 'bird' remain indeterminate, but so do claims such as that ordinarily expressed by 'Planes are birds'. This reply may satisfy the dialectical needs Chalmers has while defending the scrutability thesis, but it does not block our Wilson-

\footnotetext{
${ }^{18}$ Roughly, this is the claim that deploying one's semantic competence allows one to deduce, at any scenario $s$, any given truth at $s$ from a complete list of base truths at $s$. As per the discussion in section 3.3, this scrutability thesis is intimately related to issues surrounding the epistemology of intensions.
} 
inspired objection to E2D+CEI. Again, E2D aims to explain our knowledge of a wide range of modal truths, including those involving natural kinds, as a consequence of our a priori knowledge of intensions; this aim can be achieved only if a correspondingly wide range of intensions are determinate. ${ }^{19}$

Seizing on the imprecision of "wide range", one might reply that E2D can survive what indeterminacy there is; after all, the objector claims, even if such indeterminacy is not uncommon, it is not the norm, and crucially, is irrelevant to paradigm necessary a posteriori truths. E2D+CEI, then, can do the work it set out to do, despite such indeterminacy. We disagree. To start, we think Wilson-style cases are common. But, more importantly, they are not the only kind of cases in which application of an expression reflects factors whose influence is not antecedently encoded in the expression itself. The historical record supplies many such cases: 'acid' initially was taken to refer to only oxygenated substances, but was later applied to HCL, for theoretical reasons now largely discarded; dispute remains over whether Newtonian uses of 'mass' apply in relativistic contexts; the decision to classify whales as mammals was a controversial affair; there's the infamous resolution declassifying Pluto as a planet. In these cases, the indeterminacy was not resolved through accident, but rather, through non-demonstrative reasoning of one sort or another---typically, broadly abductive reasoning. ${ }^{20}$ So, summing up: factors influencing predicate application can involve historical accident (as in Wilson's toy case), variable psychological features (as for 'blue'), and non-demonstrative reasoning (as for 'acid', 'mass', 'mammals', and 'planet'), none of which are antecedently encoded in relevant expression themselves; hence, widespread indeterminacy.

While E2D+CEI cannot overcome this indeterminacy, it is no problem for abductive twodimensionalism. For, when considering whether to apply an expression in a given scenario, abductors can consider not only historical accident and psychological variability, but also any non-demonstrative

\footnotetext{
${ }^{19}$ In a symposium on Chalmers (2012), Chalmers (2014) suggests that he now prefers a different strategy for addressing indeterminacy, one that preserves a fully general scrutability thesis by shifting the indeterminacy at issue to scrutabilty itself. This alternative approach cannot help E2D+CEI in the present context.

${ }^{20}$ One should note that Chalmers's initial suggestion for treating Wilson's cases, by assigning distinct intensions for each decision that the natives in fact make (albeit accidentally) is no help here, since the decisions at issue in the present cases are not the result of accident. Nor does it make sense to extend Chalmers's original suggestion (repeated in Chalmers and Jackson 2001) by taking standards of non-demonstrative reasoning to be built into associated intensions, such that, e.g., if an appeal to fruitfulness pushes chemists to apply 'acid' to HCL at some scenario, then its intension includes that principle; for building abductive principles into intensions would radically multiply associated concepts, thereby eliminating most if not all substantive disagreement - and, contrary to Chalmers (2011), we see that as a serious cost.
} 
rational grounds that might push one way or another. Hence, abduction, unlike conceiving, can be productive (it is ampliative, after all), allowing those who are identifying intensions to consider how the concept should be applied, through the proper use of abduction, even when the application conditions are to some extent indeterminate. Since abduction can, in a rational way, go beyond what expressions antecedently encode, AEI has the potential to overcome broadly conceptual indeterminacy, extending applications of natural kind predicates to new scenarios or situations, on ultimately rational grounds. For example, an idealized version of a competent user of 'bird' who has never before seen an airplane would plausibly be in position to consider and compare theories of the intensions of 'bird' by attention to which theory would be, among other desiderata, most simple, unifying (e.g., of experience of flying entities), plausible, explanatorily fruitful, and so on. Similarly, for the cases of 'mass', 'Pluto', 'water', and so on. Here it is worth noting, by the way, that there is no barrier to an idealized abductor engaging in such extensions of the terms/concepts at issue, for this is more or less what language-users do as a matter of course.

\subsection{Inability to sort good from bad dispositions}

The second access-based objection is due to Melnyk (2008), who claims that E2D cannot be combined with any plausible account of concept possession/content-determination. Melnyk first argues (pp. 269271) that E2D fits poorly with externalist accounts (e.g., Millikan 1984; Fodor 1998), according to which (roughly speaking) one's possession of a concept depends on one's standing in the right relations to external entities, which relations determine the content of the concept. Melnyk next argues that E2D fits poorly with internalist accounts, according to which (roughly speaking) one's possession of a concept depends only on factors internal to the possessor, which factors determine the content of the concept.

Advocates of E2D rightly grant that externalist accounts of concept possession/content-determination are not suitably combined with E2D. ${ }^{21}$ Melnyk's objection, then, turns on whether any internalist account

\footnotetext{
${ }^{21}$ Relatedly, we maintain that the ability to use an expression deferentially is not enough for competence. Stuck in his "Chinese Room”, Searle can use '水' to refer to whatever Mandarin speakers refer to with that symbol, but Searle is not thereby competent with the associated expression.
} 
provides a better fit. Why does Melnyk think none do so? This reflects that any plausible internalist account is dispositionalist, such that "to possess a concept with a given content is (at least in part) to have a certain set of mental dispositions to use the concept, i.e., dispositions to apply the concept under certain circumstances, or to make certain inferences using it, or to do some combination of both" (p. 271). ${ }^{22}$ If a dispositionalist account were suitably combined with E2D, then competent users of a typical natural kind expression $E$ could identify its primary intension by checking their dispositions to apply $E$ at various scenarios. But competent users can, and typically do, have at least some incorrect dispositions to apply such expressions, both due to fallibility and to distance from the end of inquiry. So, a dispositionalist account is suitably combined with E2D only if competent users can determine which dispositions reflect the true nature of the expressed concept on the basis of their competence alone. Melnyk thinks this cannot be done; hence he concludes that no internalist account is suitably combined with E2D.

How can advocates of E2D reply? Given CEI, their best hope is along the following lines:

$[\mathrm{O}]$ ne can distinguish concept-constituting mental dispositions from other mental dispositions by the acceptably a priori procedure of attending to what one can conceive. Presumably one can do so in this way because a concept-constituting mental disposition just is a mental disposition that stands in a certain relation to something that one cannot conceive. Corresponding to each mental disposition is a generalization, and a disposition is a concept-constituting one iff you cannot conceive a counterexample to its corresponding generalization. Thus, imagine you're disposed to apply SWAN only to birds. Because (say) you cannot conceive of a swan that's not a bird, your disposition is a concept-constituting disposition. (Melnyk 2008, p. 276)

As Melnyk compellingly argues, however, having a disposition while being unable to conceive a counterexample to its generalization ensures neither that the disposition is central to the concept (i.e., is concept-constituting), nor that the generalization is true. His illustrative case is a chef who possesses a concept of vinegar, but incorrectly believes that vinegar is formic acid, and so finds it inconceivable that anything else be vinegar. To be sure, given complete access to the base truth at a scenario, a suitably idealized version of the chef (aware of the chemical/microphysical facts of distribution of substances in both labs and kitchens) would be able to identify an inconsistency in her dispositions to apply 'vinegar'.

\footnotetext{
${ }^{22}$ According to the main internalist alternative to dispositionalist accounts, competence with a concept $C$ requires explicitly associating $C$ with a definition consisting of necessary and sufficient conditions for its application. But as Chalmers and Jackson (2001, pp. 320-321) note, one can possess concepts (e.g., of knowledge) without being able to identify such conditions. One can see that Chalmers and Jackson moreover endorse a dispositional account when they take intuitive reactions (to hypothetical cases, conditionals, etc.) to reveal (and be necessary for revealing) a priori knowledge of concepts and relevant intensions.
} 
But---and this, we take it, is the crucial point---this inconsistency cannot be resolved by appeal to conceiving alone, since seeing the inconsistency does not show which disposition should be rejected. Moreover, incorrect dispositions need not conflict with any correct disposition---the latter may have no bearing on the former---and thus, one may have an incorrect disposition without there being a relevant inconsistency. (To make matters worse, on CEI, the sorting of dispositions into "good" and "bad" must eventuate in conclusively justified beliefs.)

One might anticipate our response (that's good, by the way: we're offering not only a better epistemology of intensions, but one that is systematic). Abductors, unlike conceivers, have resources for evaluating their dispositions. Consider the chef case. Conceiving may reveal to (an idealized version of) the chef that her dispositions to apply 'vinegar' are inconsistent. Only abduction, however, allows (an idealized version of) the chef to prioritize those dispositions, and so reject the disposition to apply 'vinegar' to formic acid. Only AEI, then, overcomes the likely fact that even at our best we are typically, to some extent, incorrectly disposed to apply our expressions/concepts. Of course, the results of abduction, such as the chef's newfound belief that vinegar is not formic acid, will not be justified conclusively, even in idealized circumstances. Fortunately, as above, restoring the desirable link between necessity and a priority requires only that the results of abduction typically track what the intensions at issue are, in fact; and here we have no reason to be pessimistic.

Let us sum up the results of this section. AEI, unlike CEI, makes room for abductive considerations to enter into rational reflection, and so, can overcome difficulties in accessing primary intensions that undermine E2D+AEI, including those surrounding indeterminacy and incorrect dispositions to apply a given term/concept (thus enabling E2D to be supplemented with a plausible and suitable account of concept-possession). Hence AEI, unlike CEI, can deliver the comprehensive access to intensions that E2D requires. Abductive two-dimensionalism is thus superior to standard conceiving-based versions, in having the resources to respond to the various access-based objections. 


\section{Contrasts in application and explanatory power}

We have argued that abduction provides the basis for effectively replying to access-based objections; for it often can deliver primary intensions where conceiving cannot. It follows that abduction and conceiving sometimes deliver different primary (and hence secondary) intensions. We now illustrate, by attention to the case that is often at the forefront of discussions of E2D---namely, the mind-body problem---how these different versions of E2D might deliver different generalized secondary intensions even given constant primary intensions. This discussion also serves to illustrate another advantage of AEI over CEI, independent of those associated with E2D---namely, that AEI makes better sense of both actual philosophical practice and disagreement.

\subsection{On the possibility of zombies: characterizing actual practice}

Zombies are minimal physical duplicates of ordinary people. They differ from us, nonetheless, in that they are not conscious. A zombie scenario is a scenario in which there are zombies; a zombie world is a world in which there are zombies. CEI implies that the primary intension of (relevant uses of) 'zombie' has a positive extension at some scenario if one can conceive of a zombie scenario, and that the secondary intension of (relevant uses of) 'zombie' has a positive extension at some world if one can conceive of a zombie world (i.e., conceive of a zombie scenario on the hypothesis that our very own world is actual). So, given E2D, CEI implies that, if one can conceive of a zombie world, then zombies are possible, which (given plausible assumptions) implies that consciousness is not physical. E2D thus implies that considerations dealing with, for example, mental causation and parsimony (so often invoked in this context to support physicalism) are not just outweighed by countervailing considerations, but are moreover irrelevant when exploring the mind-body problem.

By contrast, abductive two-dimensionalism can assign such considerations an important role. Suppose that the causal efficacy of consciousness is an explanandum for the theories at issue (i.e., the propositional representations of intensions for relevant terms, such as 'zombie' and 'pain'). Accordingly, any such theory "scores points" for accounting for, and "loses points" for precluding, mental causation. Suppose 
also that consciousness can be efficacious only if it is physical. Given these suppositions, a theory can explain the explanandum in question only if it allows that consciousness is physical. In turn, only a theory that gives (relevant uses of) 'zombie' a null extension at every world allows that consciousness is physical. As such, the latter intension can potentially explain something that an intension that gives (relevant uses of) 'zombie' a non-null extension at some world cannot. Insofar as principles of explanatory comprehensiveness are among the abductive principles, then, AEI makes room for considerations pertaining to mental causation to be relevant to exploring the mind-body problem. A similar point can be made as regards parsimony. To be sure, the final abduction will involve many principles, many explananda, and a balancing---and perhaps the theory that consciousness is not physical can explain mental causation after all, and more generally, will provide the best explanation of the explananda at issue (cf. Robinson 2016). The present point is just this: AEI makes these considerations relevant, which opens up the possibility that the intension of (relevant uses of) 'zombie' ('pain', etc.) is at odds with that which follows upon mere conceiving.

Consider, more generally, how the dispute surrounding the mind-body problem actually proceeds. Those who believe that consciousness is physical typically offer various considerations to support their belief. Even those who think that zombies are misconceived (in that the primary intension of (relevant uses of) 'zombie' has no non-null extension) rarely nakedly insist that that decides the issue. Rather, they point to considerations pertaining to, e.g., induction---e.g., that other initially mysterious phenomena have turned out to be physically explainable (cf., Smart 1959). These considerations, taken together, are supposed to count for something, regardless of what one can conceive. Whereas this practice is inexplicable given CEI, on AEI, it makes perfect sense.

\subsection{AEI and the appropriate accommodation of disagreement}

The dispute over the possibility of zombies provides concrete illustration of how abductive twodimensionalism, and more generally, AEI, accommodates disagreement among equally competent disputants. AEI makes any and all principles that might guide rational thought relevant to the 
epistemology of intensions. This methodological breadth enables AEI to treat disagreement in a way that CEI cannot. To start, as the ongoing debate over the possibility of zombies illustrates, equally competent speakers can disagree about the extension of a token expression, despite agreeing about the relevant descriptive facts (concerning, in particular, the base truth as expressed in semantically neutral language). CEI implies that such disputants are talking past one another---expressing different concepts with the same expression, a consequence that Chalmers (2011) embraces, misguidedly in our view. ${ }^{23}$ If, for example, Chalmers and Jackson disagree about the extension of (relevant uses of) 'zombie' at some scenario (and they do), then the proponent of CEI (as they are) must maintain either that at least one disputant is not thinking clearly, or that they are talking past one another. AEI, by way of contrast, allows such differences in judgments to reflect neither incompetence nor failure to recognize merely terminological disagreement, but rather, differences in disputants' views on which abductive principles are at issue, how the principles should be balanced in this case, which explananda belong to which theories/intensions, and so on. As with the dispute over the possibility of zombies, we think that some, and perhaps many, such disputes are substantive. In making such explanatory room for substantive disagreement, AEI is superior to CEI.

The role that AEI assigns to experience also helps account for substantive disagreement. As we discuss below, AEI allows that certain abductive parameters can be fixed by experience. ${ }^{24}$ Such honing will plausibly sometimes affect which extensions one who uses abduction assigns to relevant expressions; for example, which properties one is willing to inductively project may affect which extensions one assigns to a given expression. AEI thus makes room for different experiences to yield substantive disagreement about intensions. One might wonder whether CEI could replicate this advantage, either by taking concepts to tacitly contain reference to abductive principles and parameters, or by reference to the role experience

\footnotetext{
${ }^{23}$ Chalmers (2011) argues directly that disputes not expressible using basic concepts are terminological. Interestingly, since (independently of E2D) he suspects that the choice of one empirically adequate set of basic concepts over another is largely a matter of taste, it seems that there is little if any substantive disagreement, on his view of the matter. While many disputes are no doubt terminological and distinguishing substantive from terminological disputes can be quite difficult, we doubt that there is so little substantive dispute.

${ }^{24}$ To prefigure, this role for experience is relevantly like its role in concept acquisition: in both cases, experience provides certain content needed to engage in epistemic deliberation, without undermining the broadly formal or structural reasons for thinking that the products of abductive deliberation are a priori.
} 
might play in teaching conceivers to be appropriately imaginative. But neither strategy accommodates substantive disagreement. The first strategy multiples concepts, requiring a distinct concept for each distinct abductive parameter, and renders disputes involving those concepts merely verbal. As for the second, first note that given that the results of CEI are required to be conclusive, the role of experience must be the same for each competent user of a given language. In terms of a "principles and parameters" approach (see the next section for further discussion): for each language the parameter(s) may take only a single value. Hence the requirement of conclusive knowledge prevents experience from "tweaking" conceivability parameters in a way that would make room for substantive disagreement among speakers of the same language.

\section{Abduction as a priori}

One might accept that AEI and CEI deliver different results, and even that the former is superior to the latter for the reasons we have offered, and yet doubt that advocates of E2D should adopt AEI on the ground that AEI cannot deliver appropriately a priori knowledge because, as is commonly supposed, abduction is an a posteriori mode of inference. We now offer some considerations against this common supposition, and in particular we argue that abductive deliberation is as capable of delivering a priori justification as conceiving; see Biggs and Wilson (2016) for a more general and more thorough motivation for the a priority of abduction. Our discussion takes for granted that (contra, e.g., van Frassen 1980) abduction can yield knowledge, and focuses on establishing that abduction can yield a priori knowledge.

What makes knowledge a priori? To start, note that there are four ways in which experience might play a role in the course of a particular belief in $p$ coming to be justified. Experience might play a role in:

(1) acquiring the concepts that are required to entertain $p$,

(2) acquiring the evidence that is required to justify belief in $p$, 
(3) justifying belief in the epistemic value $e^{25}$ of the mode of inference inferential procedures that are required to justify belief in $p$, or

(4) acquiring the inferential procedures that are required to justify belief in $p$.

Can a belief for which experience plays an ineliminable role along one or more of (1)-(4) be justified ("entirely") a priori? The answer is: it depends on which role is at issue.

In re (1): it is commonly maintained, and advocates of CEI endorse, that belief in $p$ can be justified a priori even if experience is needed to acquire the concepts that are required to entertain $p$. For example, belief in sisters are siblings can be justified a priori even if we need experience to acquire the concepts sister and sibling. ${ }^{26}$

In re (2): it is commonly maintained that belief in $p$ cannot be justified a priori if experience is (at all) needed to acquire the evidence supporting $p$. For example, belief in water is identical with $\mathrm{H}_{2} \mathrm{O}$ (i.e., in water and $\mathrm{H}_{2} \mathrm{O}$ are a single kind) is commonly held to be justified a posteriori, on grounds that justifying it requires, in part, acquiring empirical evidence to the effect that all and only samples of water are samples of $\mathrm{H}_{2} \mathrm{O}$; hence it is that the associated modal claim (necessarily, water is $\mathrm{H}_{2} \mathrm{O}$ ) is deemed an a posteriori necessity. That said, advocates of CEI maintain, as above, that a priori knowledge of intensions provides a basis for such a posteriori knowledge: although one cannot know that water is $\mathrm{H}_{2} \mathrm{O}$ in all possible worlds without first learning through experience that the watery stuff is actually $\mathrm{H}_{2} \mathrm{O}$ (that enough of what we have called 'water' has been $\mathrm{H}_{2} \mathrm{O}$, or whatever---we ignore this complication going forward), the conditional claim that if the watery stuff is actually $\mathrm{H}_{2} \mathrm{O}$, then water is necessarily $\mathrm{H}_{2} \mathrm{O}$, falls out of relevant intensions, which are known a priori.

In re (3): it is commonly maintained, and advocates of CEI endorse, that belief in $p$ cannot be justified a priori if experience is needed to justify the belief that the inferential procedures that are required to

\footnotetext{
${ }^{25}$ An inferential procedure $m$ has epistemic value if, for any subject $s$, conclusion $c$, and premise(s) $p$ : if $s$ justifiably believes $p$ and uses $m$ to infer $c$ from $p$, then (absent defeaters) $s$ justifiably believes $c$.

${ }^{26}$ The 'can' here and in discussion of the other roles experience might play in justifying a given belief is to be understood as 'can, in the circumstances'. Belief in some claims (e.g., the four-color theorem) admit of both a priori and a posteriori justification, but our question here is not whether a belief that was justified, e.g., by means of a computer proof, can be justified in some different, a priori, fashion, but rather the question of whether, given the specific means by which the belief was justified, is the resulting justification a priori.
} 
justify belief in $p$ have epistemic value. As we will discuss shortly, it is this role for experience that is the primary basis for the common thought that abduction is not an a priori mode of inference, with the suggestion being that the epistemic value of abductive principles, hence of abduction, is an empirical matter.

In re (4): although the role of experience in an agent's acquiring or learning how to deploy a given inferential procedure is not much discussed (nor by proponents of CEI), it seems reasonable to maintain that this role is relevantly similar to that of (1): in each case experience contributes to belief (or supposition) formation, not to justification. For example, students often need encouragement to think in an appropriately imaginative way about what is possible; but that such imaginative "training up" is required in order to engage in suitably competent conceiving is not taken to undermine the status of conceiving as an a priori mode of inference. Hence it seems reasonable to maintain that belief in $p$ can be justified a priori even if experience is required in order to acquire or learn how to deploy the inferential mode at issue.

How do advocates of AEI---how do we---view the bearing of roles 1-4 on a priority? We take roughly the same stances as advocates of CEI on 1-3. Since our stances on 1-2 are identical with theirs, we say nothing more about them. Since our stance on 3 invites different dialectical burdens, we discuss it in some detail. We also discuss our stance on 4 in some detail since it may differ from theirs; since they don't discuss 4 , we cannot be sure.

What makes the dialectical burdens for CEI and AEI differ regarding 3? Advocates of CEI must show that experience need not play a role in learning that conceiving has epistemic value; we must show the same for abduction. This claim---that the epistemic value of abduction, including that of its underlying principles, can be established a priori---is uncommon. Nonetheless, it is arguably true, as we now show.

To start, for any equally explanatory theories $T$ and $T^{*}$, if $T$ scores at least as well and in some cases better than $T^{*}$ on every theoretical virtue, then choosing $T^{*}$ over $T$ would be irrational. We can thus know a priori that "counter-abduction" does not have epistemic value. Conversely, if $T$ does as well and sometimes better than competitors on every theoretical virtue, then choosing $T$ is rational. We can thus 
know a priori that abduction has epistemic value. The point extends to individual abductive principles. For any theories $T$ and $T^{*}$, if $T^{*}$ is more convoluted than $T$, and $T$ and $T^{*}$ are otherwise equal, then choosing $T^{*}$ over $T$ would be irrational. We can thus know a priori that "counter-parsimony" does not have epistemic value. Conversely, if $T$ is more parsimonious than $T^{*}$, and $T$ and $T^{*}$ are otherwise equal, then choosing $T$ over $T^{*}$ would be rational. We can thus know a priori that a principle of parsimony has epistemic value. These asymmetries are illuminated, as we see it, by the broadly transcendental supposition that abduction and its principles are constitutive of human reasoning: like principles of logical inference, the epistemic value of abduction and associated principles is a necessary precondition for the possibility of human reasoning. ${ }^{27} \mathrm{We}$ contend, then, that we can know a priori that abduction and its underlying principles have epistemic value. Though these considerations invite further discussion, they suffice to motivate our position on 3; moreover, we have here gone further toward meeting our dialectical burden in re 3 than advocates of CEI go toward meeting theirs.

One might object that following, say, counter-parsimony would be irrational only because our world happens to be parsimonious (or otherwise such that following counter-parsimony typically would lead us to falsehoods), and correspondingly following parsimony is rational only because our world happens to be parsimonious (or otherwise such that following parsimony typically leads us to truth); and so, in order to justifiably believe that following counter-parsimony would be irrational and following parsimony is rational, one must learn through experience what kind of world we occupy---and so, for any belief that is justified through abduction, experience must play a role along (3), and so, abduction cannot deliver the a priori knowledge that E2D requires. We argue below, in responding to a concern raised by Chalmers and Jackson (2001), that knowledge that our world is parsimonious plays no role in justifying belief in the epistemic value of parsimony, and what we say there generalizes to other principles. We cannot explore here the more general concern that the epistemic value of these principles hinges on our world being

\footnotetext{
${ }^{27}$ This follows Kant's (1781/1998) take on logic. The claim that abductive inference is constitutive of human reasoning is independently plausible (cf., Gelman and Markham 1983; Feeney and Heit 2007). Incidentally, we think that abduction is the ultimate arbiter of disputes about logical principles (e.g., modus ponens) and their applications as well; but that's a topic for a different paper.
} 
(contingently) such that they are truth-conducive. We do address this concern in detail elsewhere, however (Biggs and Wilson, 2016), where we argue that our justification for believing that abduction and its principles have epistemic value does not rely (and moreover cannot rely) on the belief that they are truth-conducive. Very roughly, we argue there, inter alia, that all theoretical virtues include a ceteris paribus clause, and any case in which a given abductive principle might at first appear not to be truthconducive must be a case in which other explanatory considerations were not equal.

Finally, in re 4, we maintain, as advocates of CEI may, that even if acquiring or applying an inferential procedure (as distinct from establishing its epistemic value) requires experience, its deliverances can be a priori. Why think that experience may be required here? Even if we are natural born abductors, it is plausible that experience can tweak parameters associated with abductive principles, affecting, for example, the sample size one requires for inductive generalization. Such tweaking is compatible with abduction's being an innate, and indeed necessary, component of our rational cognitive economy. Compare the principles and parameters approach to grammar (cf. Chomsky and Lasnik 1993), according to which experience gives broadly contingent content to parameters in grammatical structures that are in the relevant sense necessary, in being determined independently of experience and common among all speakers of anything we would recognize as language. Similarly, in the case of abduction, experience gives broadly contingent content to parameters in epistemic structures that are determined independently of experience, and (as suggested above) common among all thinkers engaging in anything we would recognize as right reasoning. This role for experience is relevantly like its role in concept acquisition: in both cases, experience provides certain content needed to engage in epistemic deliberation, without undermining the broadly formal or structural reasons (such as those we gave in re 3) for thinking that abductive deliberation is an a priori mode of inference.

We conclude, therefore, that the deliverances of AEI are "as a priori as" those of CEI, and so, are "a priori enough" to allow E2D to fulfill its promise. 


\subsection{Abductive principles are norms}

Do existing advocates of E2D have a ready-made reply to this claim that abduction is an a priori mode of inference? Though none explicitly consider AEI, Chalmers and Jackson come close to considering abduction as a means to identifying intensions when rejecting Block and Stalnaker's (1999) suggestion that "simplicity" might play an inescapable role in reductive analysis. Here, we extract an objection to AEI from that discussion (2001, pp. 342-350). There is, to be sure, a crucial difference between the objection to AEI that we develop, and what Chalmers and Jackson say about simplicity. The difference is inescapable because we think of abduction as involving a priori principles, while their discussion presents simplicity as an empirical fact. Indeed, it is due to this difference that their reply to Block and Stalnaker misses the mark when directed against AEI. ${ }^{28}$

As an example of an abductive principle, take the principle that one should prefer parsimonious theories. Call the following (rough) specification Parsimony: for any theories $T$ and $T^{*}$, and property $F$, if $T$ differs from $T^{*}$ only in that $T^{*}$ includes $F \mathrm{~s}$ as basic but $T$ does not, then ceteris paribus we are more justified in believing $T$. Now, given a scenario $s$, where $D$ is the base truth at $s$, and $A$ is some ordinary truth that one can infer somehow or other from $D$, the Chalmers-Jackson-inspired objection to AEI proceeds as follows. On the one hand, if $s$ is not parsimonious, Parsimony is at best irrelevant and at worst misleading when inferring $A$; hence Parsimony cannot play a role in justifying belief in $A$. On the other hand, if $s$ is parsimonious, one can derive Parsimony from $D$; hence Parsimony cannot play an essential role in justifying belief in $A$. Recall that on E2D the epistemology for inferring ordinary truths from base truths is the epistemology of intensions. So, Parsimony plays no essential justificatory role in the epistemology of intensions. Generalizing from the case of Parsimony, abduction plays no such role.

Consider first the objector's premise that Parsimony cannot play a justificatory role when assessing a scenario that is not parsimonious. We are suspicious of two aspects of this premise. First, we doubt one can make sense of a scenario's being un-parsimonious. Second, even if one can make sense of this, we

\footnotetext{
${ }^{28}$ Chalmers (2012) rehearses the Chalmers-Jackson (2001) discussion of simplicity-qua-empirical-truth, and offers nothing else that's relevant to AEI.
} 
think Parsimony can play a justificatory role when assessing un-parsimonious scenarios.

What might it be for a scenario to be un-parsimonious? Is our world un-parsimonious because it has more than one kind? How many kinds must a world have to be un-parsimonious? Two? Three? Fortytwo? These questions are silly precisely because Parsimony is a principle that guides theory choice, not a fact that holds (or not) depending on the scenario. Perhaps one can see how the proposition the world is parsimonious could be false at a complex scenario---our world is pretty complex; perhaps it is false here. But, again, we do not think of abductive principles as propositions of this sort; they are principles, norms that guide theory choice. If a scenario is un-parsimonious, then, it is not in virtue of a proposition being false at that scenario, but is rather in virtue of ... what?

Perhaps a scenario is un-parsimonious just in case, ceteris paribus, one who uses Parsimony would do worse than one who uses Anti-Parsimony: for any theories $T$ and $T^{*}$ and property $F$, if $T$ differs from $T^{*}$ only in that $T^{*}$ includes $F \mathrm{~s}$ as basic but $T$ does not, then ceteris paribus we are more justified in believing $T^{*}$. But what reason could one have to prefer Anti-Parsimony to Parsimony? Given that all other things are equal, why should one prefer a theory that includes $F \mathrm{~s}$ as basic to one that includes $F \mathrm{~s}$ as derivative? No experience could cut either way. Nor do we see any a priori consideration that might cut against Parsimony. We suspect that there is no more a scenario where one (epistemically) should follow AntiParsimony than there is a scenario where one (morally) should harm others for fun. The details of the scenario are simply irrelevant.

Suppose, nonetheless, that one can make sense of a scenario's being un-parsimonious, that $s$ is such a scenario, and that Parsimony would lead one to wrongly conclude that $A$ is false at $s$. The objector thinks that Parsimony cannot play a justificatory role when assessing $s$, since Parsimony misleads us. Recall, however, that a central goal of E2D is to explain our expressions and our use-of/reasoning-with those expressions (and associated concepts), pertaining, in particular, to their intensions and the modal profiles of their referents. Given these goals, it is natural to use our principles, even when assessing scenarios where they mislead. If assessment of scenarios need not respect the principles we actually use, how can it be relevant to how we think? Should an account of our a priori deliberation take into account the in- 
principle viability of counter-induction, anti-bivalence, and the like? Arguably, such inferential aberrations should play no more role in an account of the epistemology of intensions than should the sort of meta-semantic variations of 'tiger' (as, e.g., referring to couches) that, we presume, are irrelevant to E2D.

The second premise of the Chalmers-Jackson-inspired objection holds that Parsimony cannot play a justificatory role when assessing parsimonious scenarios. If the first premise misses the mark, so does the second; for it would be astounding if Parsimony could play a justificatory role except when assessing parsimonious scenarios! The presupposition that a scenario can be parsimonious, moreover, is no more plausible than the presupposition that a scenario can be un-parsimonious.

It is nonetheless informative to consider the second premise in more detail. According to the objector, Parsimony cannot play a justificatory role when assessing parsimonious scenarios, because one could derive the fact of their parsimony from base truths. In order for the objection to succeed, one must be able to derive "the fact" that the world is parsimonious from base truths (at parsimonious scenarios) by conceiving. This implausibly requires that (at parsimonious scenarios) it is inconceivable for the base truths to hold and the fact "the world is parsimonious" to be false. What truths might be inconceivable in conjunction with this "fact"? Not truths about the number of kinds (two, three, forty-two) that a scenario includes; for even modulo the concern that Parsimony is a principle as opposed to a fact, one can conceive that a world is parsimonious even though it includes many kinds. Moreover, even if one can derive the fact that the world is parsimonious from base truths at any parsimonious scenario through conceiving (whatever that means), perhaps Parsimony can play more than a "mediating" role in assessing such scenarios. Suppose that one uses bivalent logic to argue for multi-valued logic. Does this show that multi-valued logic plays at most a mediating role (not a justifying role) in belief formation? Plausibly not, for one who concludes that multi-valued logic should replace bivalent logic might well reassess inferences that were initially made using bivalent logic. Similarly, one who derives Parsimony from base truths at a scenario via conceiving might well use Parsimony to reassess inferences that were made by conceiving; for example, one who first uses conceiving to conclude both that mind and body are distinct 
and that Parsimony is true might then use Parsimony to reassess the inference that led to the former conclusion, perhaps concluding (through Parsimony) that mind and body are one.

Thus the Chalmers-Jackson-inspired objection to the claim that the results of abductive deliberation are a priori fails. Since Chalmers and Jackson come no closer to addressing abduction than this, their work contains no ready-made objection to abductive two-dimensionalism. One might wonder, however, whether an objection would emerge were they to clarify how conceiving works. Aside from their insistence that conceiving does not involve abductive principles, however, what little they say cuts the other way. The claim that conceiving is "rational reflection" does not preclude abduction. Nor does the intimation that conceiving is akin to "seeing" concepts; for it is likely that justification-conferring perceiving involves (perhaps tacit) abduction-e.g., knowing that there's a table in front of me, as opposed to a hallucination or a table-shaped array of sense data, plausibly requires using abduction (cf., Russell 1912/2009). Of course, one could insist that rational insight and abduction involve distinct cognitive faculties. Perhaps this un-parsimonious view would be plausible if abduction could not yield a priori knowledge of relevant intensions. But, as we have argued, abduction can yield such a priori knowledge. $^{29}$

\section{Does abduction deliver incorrect results?}

Even granting that abductive two-dimensionalism has certain advantages over E2D+CEI, one might be concerned that abduction will too often deliver intensions that are incorrect, so that conceiving must remain the ultimate route to (and arbiter of disputes about) intensions (perhaps supplemented by appeals to abduction as filling out intensions when conceiving alone falls short). This concern might be thought to beg the question against AEI, if it presupposes that abduction's deliverances are wrong whenever it and some other method (presumably, conceiving) come into conflict. Nonetheless, if abduction were to

\footnotetext{
${ }^{29}$ Though Chalmers and Jackson deny that abductive principles enter into the epistemology of intensions, their reply to Block and Stalnaker offers an alternative place for such principles, as partly constitutive of individual concepts. On this view, appeal to abductive principles in the course of considering how to apply an expression would be compatible with the outcome of the deliberation's being a priori. But as previously noted, it is a bad idea to build abductive principles into concepts, since doing so prevents there being substantive disagreement over the extensions of what are intuitively univocal expressions; see $\S 4.2$ for further relevant discussion of disagreement.
} 
frequently deliver intensions at odds with strong intuitive judgments, that would indeed be a cost.

To start: is there reason to expect abduction to frequently deliver primary intensions at odds with strong intuitive judgments? Our discussion of abduction qua guide to primary intensions provides no reason to think it will. Indeed, that discussion suggests that abduction and conceiving mostly converge vis-à-vis primary intensions because, as per footnote 13 , the explananda that serve as input to abduction given AEI are (at least typically) similar, if not equivalent, to the intuitive judgments that are crucial for determining primary intensions given CEI. Of course, this similarity does not ensure that AEI and CEI will produce the same results, precisely because abduction, being ampliative, can impact the input in ways that conceiving cannot. Nonetheless, it suggests that abduction will at most infrequently deliver primary intensions at odds with strong intuitive judgments. To be sure, as per our discussion of access-based objections to E2D, abduction will frequently deliver determinate intensions where intuition is silent, but that very silence ensures that in such cases abduction is not at odds with strong intuitive judgments. Also as per that discussion, in cases where the input (intuitions as well as, e.g., theoretical results) conflicts, abduction provides a basis for reconciling the input, sometimes by deeming some intuitive judgment false or incorrect---but that's just to be expected, and in the cases at issue, desired.

Should we expect abduction to frequently deliver secondary intensions at odds with strong intuitive judgments? Here again we think not, and below we gesture at why. We start, however, by blocking an argument that it will do so.

In discussing the intension of ordinary tokens of 'water', we suggested that a principle of parsimony, and hence perhaps abduction (assuming that other explanatory virtues are equal between the candidate intensions), supports a secondary intension for 'water' that is consistent with its being identical with $\mathrm{H}_{2} \mathrm{O}$ but not with its being (wholly) distinct from $\mathrm{H}_{2} \mathrm{O}$. In discussing zombies we suggested that a principle of parsimony, and hence perhaps abduction, supports an intension for ordinary tokens of 'pain' that is consistent with physicalism but not dualism. An objector might take these examples to imply that abduction always, or at least too often, supports the most parsimonious theory, and so will often be at odds with strong intuitive judgments. For example, since the theory that chordate and renate are the same 
kind is more parsimonious than the theory that they are (wholly) distinct kinds, the objector might suppose that abduction will deliver secondary intensions on which appropriate tokens of 'chordate' and 'renate' have the same modal extension, contra our strong intuitive judgment that these kinds are modally separable (even though actually coincident).

In response, we start by reiterating that abduction is not based only in considerations of parsimony; as such, assigning parsimony a role when choosing among competing secondary intensions does not imply that this role will be determinative of the outcome. Suppose that a theory on which relevant tokens of 'chordate' and 'renate' have distinct secondary intensions explains something better than any theory on which they have the same secondary intension. In that case, the outcome of the abductive inference will depend on how parsimony should be balanced against (perhaps inter alia) the extra explanatory power to be gained by not assigning the terms the same modal extension. To be sure, if all other explanatory considerations are equal, abduction pushes towards the most parsimonious theory, but that seems right, not problematic. If a theory on which 'chordate' and 'renate' have distinct secondary intensions offers no explanatory advantages over one on which they have the same secondary intension---if the first theory is not more systematic than the second, doesn't explain anything that the second theory can't explain, etc.--why suppose that they have distinct secondary intensions? This is tricky ground for the objector to navigate: pressing this objection requires identifying a reason $R$ to assign 'chordate' and 'renate' distinct secondary intensions such that abduction would not suggest that these terms have distinct secondary intensions when it takes $R$ into account, as abduction would be bound to do.

The objector, then, must identify a reason that suggests that relevant tokens of 'chordate' and 'renate' have distinct secondary intensions but that cannot itself be incorporated into abduction in a way that would lead abduction to deliver different modal extensions for these terms. Here, the objector might appeal to conceivability itself, saying, "Our ability to conceive of chordates that are not renates provides our only reason to think that chordate and renate are distinct kinds, and hence that relevant uses of 'chordate' and 'renate' have distinct secondary intensions. Given AEI, this reason cannot trump the dictates of parsimony, since otherwise AEI would fail to be distinguished from CEI. So, abduction, 
contrary to strong intuitive judgment, must suggest that 'chordate' and 'renate' have the same modal extension." We offer two independent responses to this proposal.

First, and most importantly, we think that our confidence that abduction tracks the metaphysical structure of the world should be greater than our confidence in any single intuitive judgment (supported by no further considerations), however strong it may be. Accordingly, if the conceivability of a chordate that is not a renate provides our only reason to think that chordate and renate are distinct kinds, and abductive principles suggest that they are the same kind, then, we claim, we should follow abduction and conclude that they are the same kind. The objector might respond that cases in which conceivability and only conceivability suggests that entities are distinct are common, and we should weigh our confidence that abduction tracks the metaphysical structure of the world against our confidence that at least most of the strong intuitive judgments that reflect such conceivability gets things right. But we see no antecedent reason to think that such cases are common. Indeed, we think that the chordate-renate case is recycled so often because such cases are rare.

Second, the objector's paradigm case (chordate-renate) should be controversial. Suppose that the narrow functional roles of hearts and kidneys (respectively, roughly pumping blood and filtering blood) do not determine their respective essences, but rather, their place within a larger blood servicing system determines their respective essences, such that part of the essence of hearts is to be connected in the right way to kidneys and vice versa. Given this supposition, chordate and renate are modally inseparable, and correspondingly, relevant tokens of 'chordate' and 'renate' have the same modal extension, and so, the objection fails. ${ }^{30}$ Suppose, instead, that the narrow functional roles of hearts and kidneys determine their respective essences. Given this supposition, we can model (nomologically possible) chordates that are not renates, and so, the bare conceivability of chordates that are not renates does not provide our only reason

\footnotetext{
${ }^{30}$ Two views of the nature of chordates and renates are compatible with this result. On one, hearts and kidneys are necessarily connected because they are different components of a single kind, and so, chordate and renate are the same kind---a principle of parsimony supports this view. On the other, hearts and kidneys are necessarily connected even though they are distinct existences, and so, chordate and renate are distinct kinds even though they are inseparable (and correspondingly, even though relevant tokens of 'chordate' and 'renate' have the same modal extension). Of course, this may violate Hume's Dictum--according to which there are no necessary connections between distinct existences---but we do not see this as a serious cost (see Wilson 2010 for discussion).
} 
to think that chordate and renate are modally separable, and so, the objection fails. ${ }^{31}$ Of course, one might deny that either broad or narrow functional role determines the essences of hearts and kidneys. These considerations challenge, nonetheless, the objector's premise that abduction produces results clearly at odds with strong intuitive judgment in the chordate-renate case.

We have suggested that there is no clear reason to expect that abduction will frequently deliver secondary intensions at odds with strong intuitive judgments. We now gesture at two reasons to think that, on the contrary, abduction will frequently deliver secondary intensions in conformity with such judgments, each of which requires more exploration than we can offer here. First, plausibly, abduction typically tracks strong intuitive judgments vis-à-vis secondary intensions because such judgments stem from tacit abduction (cf. Biggs 2011)---though, because various factors (e.g., cognitive biases) may impact tacit abduction in ways in which they do not impact explicit abduction, abduction will not perfectly track strong intuitive judgments. Second, plausibly, abduction will contradict intuitive judgments mostly when they are not especially strong, because intuitive judgments are especially strong when they are part of a network of mutually reinforcing considerations (many of which are broadly theoretical) that support the content of the intuitive judgment, and in such cases abduction typically will support that same content.

\section{Conclusion}

We have argued that abductive two-dimensionalism---E2D+AEI---has two important advantages over E2D+CEI. First, as compared to conceiving (understood as per CEI), abduction can deliver a wider range of primary (and hence secondary) intensions, and so provide a basis for knowledge of a wider range of modal truths, including those about natural kinds. This result is crucial for realizing the aim and promise of E2D as explaining our knowledge of a wide range of modal truths (especially those involving natural

\footnotetext{
${ }^{31}$ For example, given this supposition, we can build a model of a (nomologically possible) creature that has one organ for pumping blood and no organ for filtering blood because whenever the blood becomes so full of waste and excess fluid that the blood-pumping organ cannot use it, it acquires new blood (think vampires) and excretes the old, or a creature that has one organ for pumping blood, but for which the filtering functions that are served by the kidneys in actual mammals are instead distributed throughout various tissues that serve multiple functions, and which collectively have no claim to being a kidney.
} 
kinds) as a consequence of our knowledge of corresponding intensions (including those of natural kind terms). Second, abduction can explain actual practice surrounding philosophical theorizing (and correspondingly choice of secondary intensions) without either implying that widespread philosophical practice is nonsensical, or trivializing most philosophical disagreement as non-substantive. In each case, abduction's advantage results from it being methodologically broad, incorporating any theoretical virtues there may be, by way of positive contrast with conceiving, which is methodologically narrow, incorporating only intuitive judgments (or something relevantly similar).

We also have considered and rejected two prima facie tempting objections to abductive twodimensionalism. The first objection is that abduction cannot deliver the specifically a priori knowledge that E2D requires, since abduction, being an a posteriori mode of inference, cannot deliver a priori knowledge. In response (and drawing on our 2016), we have argued that, contrary to common opinion, abduction is an a priori mode of inference, and in particular that abduction is as a priori as conceiving. The second objection is that abduction will too often deliver results that are "wrong", in being at odds with strong intuitive judgments. In response, we have noted that contradicting strong intuitive judgment does not in itself constitute delivering the wrong result, we have argued that we have no reason to believe that abduction will frequently contradict especially strong intuitive judgments, and we have sketched reasons for thinking that, on the contrary, abduction will typically preserve strong intuitive judgments about both primary and secondary intensions, notwithstanding departures from such judgments inspired by incompatible dispositions or other input into theorizing about intensions (as in Melnyk's chef case) or appropriate attention to the full range of relevant explanatory considerations (as perhaps may be in the case of zombies).

We find, then, that abductive two-dimensionalism is superior to E2D+CEI, and more generally, is an attractive approach to meaning and modality, as per the E2D strategy.

The significance of AEI, moreover, goes beyond that of abductive two-dimensionalism, and indeed should be of interest to anyone concerned with the question of whether semantic or conceptual analysis plays a crucial role in philosophical theorizing---that is, in arriving at philosophical truth, constituting 
philosophical practice, and/or explaining philosophical disagreement. On the one hand, if one does take such analysis to play such a crucial role, then even if one rejects E2D, one's view will likely be subject to analogues of the access-based objections to E2D, undermining the basis for systematically treating philosophical truth, practice, and/or disagreement; but if such analysis proceeds via abduction (understood as per AEI), analogues of our responses to the access-based objections to E2D can be fruitfully brought to bear. On the other hand, many who deny that semantic or conceptual analysis plays a crucial role vis-a-vis philosophical theorizing do so primarily on grounds that such analysis is inadequate to play this role, as is supposedly illustrated by the need to appeal to abduction in order, e.g., to construct or choose between competing philosophical theories. If such analysis must proceed by way of conceiving, then this line of thought is probative; but if such analysis can proceed by way of abduction, then this line of thought becomes a non sequitur. Accordingly, both those who accept and those who deny that semantic and conceptual analysis plays a crucial role in philosophical theorizing should be interested in our proposal.

AEI is not only the best epistemology for E2D, but moreover, and more generally, represents the basis for a new and promising account of semantic and conceptual analysis.

\section{References}

Biggs, Stephen. 2011. 'Abduction and Modality'. Philosophy and Phenomenological Research 83: 283326.

Biggs, Stephen, and Wilson, Jessica. 2016. 'The A Priority of Abduction'. Philosophical Studies 174: 735-758.

Bealer, George. 2002. Modal Epistemology and the Rationalist Renaissance'. In Conceivability and Possibility, eds. T. Szabo Gendler and J. Hawthorne. New York: Oxford University Press.

Block, Ned, and Stalnaker, Robert. 1999. 'Conceptual Analysis, Dualism, and the Explanatory Gap'. Philosophical Review 108:1-46.

Braddon-Mitchell, David. 2004. 'Masters of Our Meaning'. Philosophical Studies 188:133-52.

Byrne, Alex and Pryor, Jim. 2006. 'Bad Intensions'. In Two-Dimensional Semantics: Foundations and Applications, eds. M. Garcia-Carpintero and J. Macià. New York: Oxford University Press.

Campos, Daniel. 2011. 'On the Distinction between Peirce's Abduction and Lipton's Inference to the Best Explanation', Synthese 180/3: 419-442. 
Carnap, Rudolph. 1947/1956. Meaning and Necessity, 2nd Edition. Chicago: University of Chicago Press.

Chalmers, David. 1996. The Conscious Mind: In Search of a Fundamental Theory. New York: Oxford University Press.

---2002. 'Does Conceivability Entail Possibility'. In Conceivability and Possibility, eds. Tamar Gendler and John Hawthorne. Oxford: Oxford University Press.

--2005. 'The Matrix as Metaphysics'. In Philosophers Explore the Matrix, ed. C. Grau. New York: Oxford University Press.

---2006. ‘The Foundations of Two-Dimensional Semantics'. In Two-Dimensional Semantics: Foundations and Applications, eds. M. Garcia-Carpintero and J. Macià. New York: Oxford University Press.

---2006a. 'Two-Dimensional Semantics'. In Oxford Handbook of the Philosophy of Language, eds. E. Lepore and B. Smith. New York: Oxford University Press.

---2006b. 'Scott Soames' E2D'. American Philosophical Association, Central Division Meeting, available at http://consc.net/papers/soamesE2D.pdf.

---2011. 'Verbal Disputes'. Philosophical Review 120: 515-566.

---2012. Constructing the World. New York: Oxford University Press.

---2014. 'Intensions and Indeterminacy: Reply to Soames, Turner, and Wilson'. Philosophy and Phenomenological Research 89: 249-269.

Chalmers, David and Jackson, Frank. 2001. 'Conceptual Analysis and Reductive Explanation'. Philosophical Review 110: 315-360.

Chomsky, Noam and Lasknik, Howard 1993. 'Principles and Parameters Theory'. In Syntax: An International Handbook of Contemporary Research.

Davies, Martin. \& Humberstone, Lloyd. 1981. ‘Two Notions of Necessity'. Philosophical Studies 58:130.

Feeney, Aidan and Heit, Evan 2007. Inductive Reasoning: Experimental, Computational, and Developmental Approaches. Cambridge: Cambridge University Press.

Fodor, Jerry. 1998. Concepts: Where Cognitive Science Went Wrong. New York: Oxford University Press.

Frege, Gottlieb. 1918/1997. 'Thought'. In The Frege Reader, ed. M. Beaney. Malden, MA: Blackwell.

Gelman, S. A. and Markman, E. M. 1986. 'Categories and Induction in Young Children'. Cognitioni 23: 183-209.

Gertler, Brie. 2002. 'Explanatory Reduction, Conceptual Analysis, and Conceivability Arguments about the Mind'. Nous 36: 22-49. 
Jackson, Frank. 1998. From Metaphysics to Ethics: A Defence of Conceptual Analysis. Oxford: Oxford University Press.

---2004. 'Why We Need A-Intensions'. Philosophical Studies 118: 257-277.

Kant, Immanuel. 1781/1998. Critique of Pure Reason. In Critique of Pure Reason, trans. P. Guyer and A. Wood. New York: Cambridge University Press.

Kaplan, David. 1979. 'Dthat'. In Syntax and Semantics. P. Cole, ed. New York: Academic Press.

---1989. 'Demonstratives'. In Themes from Kaplan. J. Almog, J. Perry, and H. Wettstein, eds. Oxford: Oxford University Press.

Kripke, Saul. 1972/1980. Naming and Necessity. Princeton: Princeton University Press.

Lipton, Peter. 1991/2004. Inference to the Best Explanation, 2nd edition. New York: Routledge.

Lewis, David. 1994. 'Reduction of mind'. In Companion to the Philosophy of Mind, ed. S. Guttenplan. Oxford: Blackwell.

Mackonis, Adolfas 2013. 'Inference to the Best Explanation, Coherence, and Other Explanatory Virtues', Synthese 190/6: 975-995.

Melnyk, Andrew. 2008. 'Conceptual and Linguistic Analysis: A Two-Step Program'. Noûs 42: 267-291.

Millikan, Ruth. 1984. Language, Thought, and Other Biological Categories: New Foundations for Realism. Cambridge: MIT Press.

Minnameier, Gerhard 2004. 'Peirce-Suit of Truth: Why Inference to the Best Explanation and Abduction Ought Not be Confused', Erkenntnis 60/1: 75-105.

Peacocke, Christopher. 1993. 'How Are A Priori Truths Possible?', European Journal of Philosophy 1: 175-99.

Perry, John. 2001. Reference and Reflexivity. Stanford: Center for Study of Language and Information Publications.

Plutynski, Anya 2011. 'Four Problems of Abduction: A Brief History', Hopos: The Journal of the International Society for the History of Philosophy of Science 1/2: 227-248.

Raffman, Diana. 1994. 'Vagueness Without Paradox'. Philosophical Review 103(1).

Robinson, William. 2016. 'Dualism without Conceivability Arguments', (abstract/program: 63), presented at Toward a Science of Consciousness, Tucson, AZ, 26 April 2016.

Russell, Bertrand. 1912/2009. The Problems of Philosophy. Seven Treasures Publications.

Smart, J.J.C. 1959. 'Sensations and Brain Processes'. Philosophical Review 68: 141-156.

Soames, Scott. 2005. Reference and Description: The Case against E2D. Princeton: Princeton University Press.

Stalnaker, Robert. 1978. 'Assertion'. In Syntax and Semantics. P. Cole, ed. New York: Academic Press. 
---1999. Context and Content: Essays on Intentionality in Speech and Thought. New York: Oxfrod University Press.

---2001. 'On Considering a Possible World as Actual'. Proceedings of the Aristotelian Society, Supplementary Volume 75: 141-156.

van Frassen, Bass. 1980. The Scientific Image. New York: Oxford University Press.

Wilson, Jessica. 2010. 'What Is Hume’s Dictum and Why Believe It?'. Philosophy and Phenomenological Research 80: 595-637.

Wilson, Mark. 1982. 'Predicate Meets Property'. The Philosophical Review 91: 549-589.

---2006. Wandering Significance: An Essay on Conceptual Behavior. New York: Oxford University Press.

Wong, Kai-Yee. 1996. 'Sentence-relativity and the Necessary A Posteriori'. Philosophical Studies 83: 53-91. 